\title{
STONE-ČECH COMPACTIFICATIONS VIA ADJUNCTIONS
}

\author{
R. C. WALKER
}

\begin{abstract}
The Stone-Čech compactification of a space $X$ is described by adjoining to $X$ continuous images of the Stone-Čech growths of a complementary pair of subspaces of $X$. The compactification of an example of Potoczny from $[\mathbf{P}]$ is described in detail.
\end{abstract}

The Stone-Čech compactification of a completely regular space $X$ is a compact Hausdorff space $\beta X$ in which $X$ is dense and $C^{*}$-embedded, i.e. every bounded real-valued mapping on $X$ extends to $\beta X$. Here we describe $\beta X$ in terms of the Stone-Čech compactification of one or more subspaces by utilizing adjunctions and completely regular reflections. All spaces mentioned will be presumed to be completely regular.

If $A$ is a closed subspace of $X$ and $f$ maps $A$ into $Y$, then the adjunction space $X \cup_{f} Y$ is the quotient space of the topological sum $X \oplus Y$ obtained by identifying each point of $A$ with its image in $Y$. We modify this standard definition by allowing $A$ to be an arbitrary subspace of $X$ and by requiring $f$ to be a $C^{*}$-embedding of $A$ into $Y$.

The completely regular reflection of an arbitrary space $Y$ is a completely regular space $\rho Y$ which is a continuous image of $Y$ and is such that any realvalued mapping on $Y$ factors uniquely through $\rho Y$. The underlying set of $\rho Y$ is obtained by identifying two points of $Y$ if they are not separated by some real-valued mapping on $Y$. The resulting set has the property that for each real-valued mapping $f$ on $Y$, a unique real-valued function $\rho(f)$ can be defined on $\rho Y$ that factors $f$ through $\rho Y$. The topology on $\rho Y$ is taken to be the weakest topology so that all of the functions $\rho(f)$ so obtained are continuous.

Lemma 1. If $A$ is a subspace of $X$ and $f$ is $a C^{*}$-embedding of $A$ into $Y$, then $X$ is $C^{*}$-embedded in $\rho\left(X \cup \cup_{f} Y\right)$.

Proof. The mappings required in the proof are illustrated in the diagram. The mappings $p_{1}$ and $p_{2}$ are the compositions of the quotient map on $X \oplus Y$ with the embeddings of $X$ and $Y$ into $X \oplus Y$ and $k$ is any real-valued mapping on $X$. We show that both $p_{2}$ and $\rho \mid p_{2}[X]$ are embeddings. Since $f$ is an embedding, $p_{2}$ is one-to-one. To show that $p_{2}$ is open onto its range, it is

Received by the editors March 13, 1975.

AMS (MOS) subject classifications (1970). Primary 54B17, 54D35; Secondary 54D60.

Key words and phrases. Stone-Čech compactification, adjunction, Hewitt-Nachbin realcompactification. 
sufficient to show that the image of a cozero-set of $X$ is a cozero-set of $p_{2}[X]$. Since $A$ is $C^{*}$-embedded in $Y$, if $k$ is any bounded, real-valued mapping on $X$, $k \mid A$ has an extension $l$ to $Y$. It then follows from the construction of $X \cup_{f} Y$ that a mapping $g$ exists so that $g \circ p_{2}=k$. Hence, the image of the cozero-set of $k$ is the trace on $p_{2}[X]$ of the cozero-set of $g$, and $p_{2}$ is not only an embedding, but is additionally a $C^{*}$-embedding.

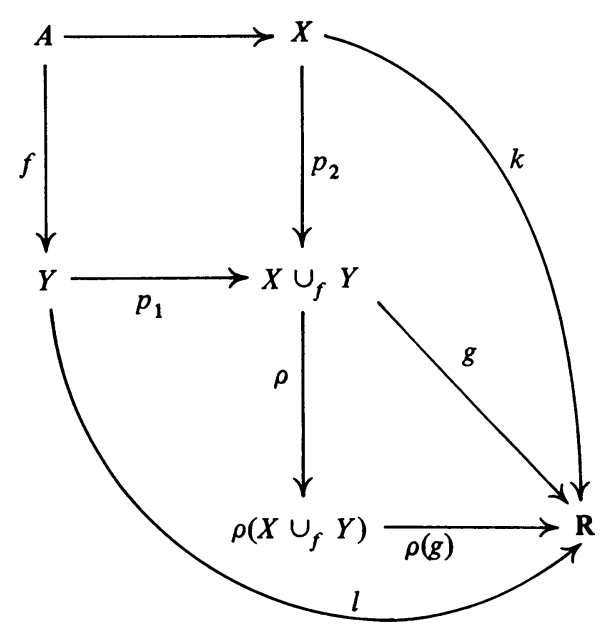

Since any two points of $p_{2}[X]$ are separated by a mapping such as $g$, their images in $\rho\left(X \cup_{f} Y\right)$ are separated by $\rho(g)$ so that $\rho$ is one-to-one on $p_{2}[X]$. In addition, $\rho \mid p_{2}[X]$ is seen to send cozero-sets to cozero-sets in exactly the same manner as for $p_{2}$. Since $\rho(g) \circ \rho \circ p_{2}=k$, we have shown that $X$ is $C^{*}$ embedded in $\rho\left(X \cup_{f} Y\right)$.

We will always take the embedding $f$ in the lemma to be the embedding $\eta_{A}: A \rightarrow \beta A$. To shorten notation, we write $(X \mid \beta A)$ for $X \cup_{\eta_{A}} \beta A$.

THEOREM 1. If $A$ is a closed subspace of $X$ such that every noncompact closed set of $X$ meets $A$, then $\beta X=\rho(X \mid \beta A)$.

Proof. From the lemma, $X$ is $C^{*}$-embedded in $\rho(X \mid \beta A)$ and $X$ is easily seen to be dense in $\rho(X \mid \beta A)$ since $A$ is dense in $\beta A$. We will show that $\rho(X \mid \beta A)$ is compact by showing that $(X \mid \beta A)$ is compact. Let $Q$ be an ultrafilter on $(X \mid \beta A)$. If any closed subset belonging to $Q$ is contained in $X \backslash A$, then the hypothesis on $A$ shows that $\mathscr{Q}$ contains a compact set and therefore converges. If no closed member of $A$ is contained in $X \backslash A$, then the family $\{(\operatorname{cl} S) \cap \beta A$ : $S \in \mathcal{Q}\}$ is a filter on $\beta A$ and therefore clusters to a point in $\beta A$. Hence, $(X \mid \beta A)$ is compact and therefore its continuous image $\rho(X \mid \beta A)$ is also.

EXAMPLE 1. The space $\Psi$ described in Exercise 5I of [G-J] is one interesting example where Theorem 1 applies. Construction of $\Psi$ begins by obtaining a maximal, infinite, almost disjoint family $\mathscr{E}$ of infinite subsets of the countable discrete space $\mathbf{N}$. A point is added to $\mathbf{N}$ for each $E$ in $\mathcal{E}$ with neighborhoods of the added point being required to contain all but finitely many points of $E$. The set of added points is taken to be the closed subspace $A$ in the theorem. Since $|A|=c,|\beta A|=2^{2^{c}}$. However, $\Psi$ is separable, so that $|\beta \Psi|=2^{c}$. 
Hence, the formation of $\rho(X \mid \beta A)$ must identify points. This is to be expected, since $\Psi$ fails to be normal, making it unlikely that the adjunction space $(X \mid \beta A)$ is Hausdorff. Since the proof of Theorem 1 shows $(X \mid \beta A)$ to be compact, we see that the operation of forming $\rho(X \mid \beta A)$ is simply one of identifying points to make the compactification Hausdorff.

The theorem also applies to the more complex space described by Burke in [B]. That space is constructed along the lines of $\Psi$, with a countable product of two point discrete spaces replacing $\mathbf{N}$.

The restriction on $A$ limits the application of Theorem 1. By using Lemma 1 twice, a more general result is obtained.

Theorem 2. If $A$ is any closed subspace of $X, \beta X=\rho(\rho(X \mid \beta A) \mid \beta(X \backslash A))$.

Proof. Applying Lemma 1 twice, we see that $X$ is $C^{*}$-embedded in $\rho(X \mid \beta A)$ and that $\rho(X \mid \beta A)$ is in turn $C^{*}$-embedded in $\rho(\rho(X \mid \beta A) \mid \beta(X \backslash A))$. The density of $X$ follows easily from that of $A$ in $\beta A$ and $X \backslash A$ in $\beta(X \backslash A)$. To show compactness, let $\mathcal{Q}$ be an ultrafilter on $(\rho(X \mid \beta A) \mid \beta(X \backslash A))$. Since $थ$ contains either $\rho(\beta A)$ or $\beta(X \backslash A)$, थ must converge. Hence, $(\rho(X \mid \beta A) \mid \beta(X \backslash A))$ and its continuous image $\rho(\rho(X \mid \beta A) \mid \beta(X \backslash A))$ are compact.

Example 2. To illustrate the theorem, we first consider $\mathbf{R}$ with $A=[0, \infty)$. The first adjunction $\rho(\mathbf{R} \mid \beta A)$ adds the "right end" of $\beta \mathbf{R} . \beta(\mathbf{R} \backslash A)$ is a copy of $\beta \mathbf{R}$, and in the formation of the second adjunction, the points of the right end of $\beta(\mathbf{R} \backslash A)$ are all identified with 0 .

EXAMPLE 3. Using Theorem 2, the Stone-Čech compactification of the example given by Potoczny can be described. Following the notation of $[\mathbf{P}]$, let $W=\left\{\lambda: \lambda<\omega_{1}\right\}$ denote the set of countable ordinals and let $T$ $=\left\{(\gamma, \lambda): 0 \leqslant \gamma<\lambda<\omega_{1}\right\}$. Define a topology on $X=W \cup T$ as follows: Points of $T$ are isolated and $V$ is a neighborhood of a point $\sigma$ of $W$ if $V$ contains $\sigma$ and all but finitely many points of the set $T_{\sigma} \equiv\{(\sigma, \lambda): \lambda>\sigma\}$ $\cup\{(\lambda, \sigma): \lambda<\sigma\}$. It follows easily that $X$ is Hausdorff, has a base of clopen sets, is locally compact, and completely regular.

We describe $\beta X$ by examining the two adjunction steps indicated by Theorem 2 where $W$ is taken to be $A$. Since $W$ is a discrete subspace of cardinality $\aleph_{1},|\beta W|=2^{2^{\kappa_{1}}}$. We will show that in the formation of $\rho(X \mid \beta W)$, all of the points of the "growth" $W^{*}=\beta W \backslash W$ are identified. The following key property of $X$ was demonstrated in $[\mathbf{P}]$ to show that $X$ is not even weakly normal:

(a) If $F$ is a countably infinite subset of $W, E$ is an un uncountable subset of $W$, and $U$ and $V$ are open subsets of $X$ containing $F$ and $E$, respectively, then $U \cap V \neq \varnothing$.

A uniform ultrafilter on an infinite set of cardinality $\eta$ is an ultrafilter whose every member also has cardinality $\eta$. In $[\mathbf{H}]$, Hindman shows that such a set admits $2^{2^{\eta}}$ uniform ultrafilters. For a point $p$ belonging to the growth of a discrete space $D$ let $A^{p}$ denote the corresponding free ultrafilter on $D$. We now show that:

(b) If $p$ is any point of $W^{*}$ and $q$ is any point of $W^{*}$ corresponding to a uniform ultrafilter, then $p$ and $q$ are identified in $\rho(X \mid \beta W)$ : Since $\rho(X \mid \beta W)$ is Hausdorff, $p$ and $q$ must be identified if they fail to have disjoint neighborhoods in $(X \mid \beta W)$. The traces on $X$ of such a pair of neighborhoods must 
contain disjoint members $P$ and $Q$ of $A^{p}$ and $A^{q}$, respectively, as subsets of $W$. Let $F$ be any countable subset of $P$ and let $E=Q$. Then $F$ and $E$ satisfy the conditions of (a), and thus are not contained in disjoint open subsets of $X$. Hence, $p$ and $q$ cannot have disjoint neighborhoods in $(X \mid \beta W)$, and are identified in $\rho(X \mid \beta W)$.

Thus, the formation of $\rho(X \mid \beta W)$ adds only a single point to $X$, call it $\infty$. Since the pre-image in $(X \mid \beta W)$ of a neighborhood $U$ of $\infty$ must be a neighborhood of every point of $W^{*}, U$ must contain all but finitely many points of $W$ together with a neighborhood in $X$ of each point of $W$ included. Hence, $U$ must also include all but finitely many points of $T_{\sigma}$ for all but finitely many $\sigma$ 's in $W$. Call such a subset of $T$ doubly cofinite. The construction of $\beta X$ is completed by adjoining $\beta T$ to $\rho(X \mid \beta W)$ and taking the reflection. In order to describe the identification of points which occurs in taking the reflection of $(\rho(X \mid \beta W) \mid \beta T)$, we first classify the free ultrafilters on $T$, and therefore the points of $T^{*}$, into three types. Let $p$ belong to $T^{*}$ and let $A^{p}$ be the corresponding free ultrafilter on $T$. Then we classify $A^{p}$ as follows:

Type I: $A^{p}$ contains a member $Z$ such that $\left|Z \cap T_{\sigma}\right|<\aleph_{0}$ for all $\sigma$ in $W$.

Such ultrafilters must exist since any ultrafilter which contains the set $\left\{(\sigma, \sigma+1): \sigma<\omega_{1}\right\}$ is of this type.

Type II: $A^{p}$ is not of Type I and $A^{p}$ contains a member $Z$ such that $\left|Z \cap T_{\sigma}\right| \geqslant \aleph_{0}$ for only finitely many $\sigma$ in $W$.

Ultrafilters of this type must exist since any ultrafilter containing $T_{\sigma}$ for some $\sigma$ has this property.

Type III: $A^{p}$ is not of either Type I or II, i.e. for every $Z$ in $A^{p}$, $\left|Z \cap T_{\sigma}\right| \geqslant \aleph_{0}$ for infinitely many $\sigma$ in $W$.

The existence of Type III ultrafilters follows from the following result found in $[\mathbf{H}]$ :

LEMMA 2. If an infinite collection $\mathbb{Q}$ of subsets of the infinite discrete space $D$ of cardinality $\eta$ satisfies:

(1) $|A|=\eta$ for all $A$ in $\mathbb{Q}$, and

(2) $\left|A_{1} \cap A_{2}\right|<\eta$ for $A_{1}$ and $A_{2}$ distinct members of $\mathbb{Q}$,

then there exists a uniform ultrafilter $A^{p}$ on $D$ such that for each $Z$ in $\left.A^{p},|A \in \mathbb{Q}:| Z \cap A \mid=\eta\right\}|=| \mathbb{Q} \mid$.

Applying the lemma to the family $\mathbb{Q}=\left\{T_{\sigma}: \sigma<\omega_{1}\right\}$ shows the existence of ultrafilters of Type III.

The description of $\beta X$ is completed by describing the identifications which take place in forming $\rho(\rho(X \mid \beta W) \mid \beta T)$. From the proof of Theorem 2, $(\rho(X \mid \beta W) \mid \beta T)$ is compact, so that $\rho$ is actually the quotient map which identifies pairs of points which are not separated by open sets. If $A^{p}$ is of Type $\mathrm{I}$, then a straightforward case-by-case argument shows that $p$ can be separated from any other point of $\rho(X \mid \beta W)$ or $T^{*}$ by disjoint neighborhoods, so that such points are not identified with any other point.

If $A^{p}$ is of Type II, then there is a member $Z$ of $A^{p}$ and a finite subset $F$ of $W$ such that $\left|Z \cap T_{\sigma}\right| \geqslant \aleph_{0}$ only for $\sigma$ in $F$. Thus, we can write $Z$ as follows:

$$
Z=\left(\cup\left\{Z \cap T_{\sigma}: \sigma \in F\right\}\right) \cup\left(Z \backslash \cup\left\{T_{\sigma}: \sigma \in F\right\}\right) .
$$

The set $Z \backslash \cup\left\{T_{\sigma}: \sigma \in F\right\}$ cannot belong to $A^{p}$ since $A^{p}$ is not of Type I. 
Hence $\cup\left\{Z \cap T_{\sigma}: \sigma \in F\right\}$ is in $A^{p}$. Therefore, $Z \cap T_{\sigma}$ belongs to $A^{p}$ for some $\sigma_{0}$. Thus, $p$ cannot be separated from $\sigma_{0}$. Since it is easily seen that $p$ can be separated from any other point, $p$ is identified with $\sigma_{0}$ in $\rho((X \mid \beta W) \mid \beta T)$.

Finally, if $A^{p}$ is of Type III, every member of $A^{p}$ meets $T_{\sigma}$ in an infinite set for infinitely many $\sigma$ in $W$. Hence, $p$ cannot be separated from $\infty$ by disjoint open sets. However, $p$ can be separated from each $\sigma$ in $W$ since for any $Z$ in $A^{p}$, the set $Z \backslash T_{\sigma}$ must belong to $A^{p}$. Therefore, $p$ is identified with $\infty$.

To complete the description of $\beta X$, it remains to describe the neighborhoods of the Type I points and of $\infty$. If $A^{p}$ is of Type $I$, then $A^{p}$ contains sets which are clopen in $\rho(X \mid \beta W)$ and can include only Type I points in their closures in $\beta T$. Hence, a basic neighborhood of $p$ in $\beta X$ is identical with a basic neighborhood of $p$ in $\beta T$. We have already seen that a neighborhood of $\infty$ in $\rho(X \mid \beta W)$ must contain all but finitely many points of $W$ together with a doubly cofinite subset of $T$. Since every Type III point is identified with. $\infty$, the pre-image in $(\rho(X \mid \beta W) \mid \beta T)$ of any neighborhood of $\infty$ must include a neighborhood of every Type III point. To relate Type III points to doubly cofinite subsets of $T$, we make the following observation:

(c) If $S$ is a subset of $T$, then every Type III point is contained in $\mathrm{cl}_{\beta T} S$ if and only if $S$ is doubly cofinite: We prove the contrapositives. If $\operatorname{cl}_{\beta T} S$ fails to contain a Type III point $p$, then $T \backslash S$ belongs to $A^{p}$ and must have an infinite intersection with infinitely many of the $T_{\sigma}$ 's. Hence, $S$ is not doubly cofinite. Conversely, if $S$ fails to be doubly cofinite, then $N_{\sigma}=(T \backslash S) \cap T_{\sigma}$ is infinite for all $\sigma$ belonging to an infinite index set $I$. By applying Lemma 2 to the family $\left\{N_{\sigma}: \sigma \in I\right\}$, we obtain an ultrafilter on $\bigcup\left\{N_{\sigma}: \sigma \in I\right\}$ which can be extended to a Type III ultrafilter $A^{p}$ on $T$. Since $A^{p}$ contains $T \backslash S, p$ has a $\beta T$-neighborhood which misses $S$.

This leads to the following property.

(d) The point $\infty$ has a clopen neighborhood base in $\beta X$ : Let $U$ be any closed $\beta X$-neighborhood of $\infty$. Then the set $F=W \backslash \operatorname{int}(U)$ is finite. Put $S$ $=\left(U \backslash\left(\cup\left\{T_{\sigma}: \sigma \in F\right\}\right)\right) \cap T$. Then $S$ is doubly cofinite, and $\operatorname{cl}_{X} S=S$ $\cup(W \backslash F)$ is clopen in $X$ and contained in $U$. Hence, $\operatorname{cl}_{\beta X} S=\operatorname{cl}_{\beta X}\left(\operatorname{cl}_{X} S\right)$ is a clopen subset of $\beta X$, contains $\infty$, and is a subset of $U$. Hence, $\infty$ has a clopen base in $\beta X$.

Since it is easy to see that every other point of $\beta X$ has a clopen neighborhood base, we have shown that

(e) $\beta X$ is zero-dimensional, or equivalently, $X$ is strongly zero-dimensional:

Here, by strongly zero-dimensional, we mean that disjoint zero-sets of $X$ are separated by clopen sets. Since this is precisely the class of spaces for which the 2-compactification and the Stone-Čech compactification coincide, we have $\zeta X=\beta X$. Finally, we describe the Hewitt-Nachbin realcompactification $v X$ of $X$.

(f) $v X=\rho(X \mid \beta W)$ : The inclusion of $\infty$ in $v X$ follows from the observations that every $G_{\delta}$ containing $\infty$ meets $X$ and that $v X$ consists of those points of $\beta X$ which cannot be separated from $X$ by $G_{\delta}$ 's. The exclusion of Type I points follows from the fact that no realcompact space can be $C$-embedded and dense in a large space. Since for each Type I point $p, A^{p}$ includes a $C$ embedded, discrete subspace of $X, p$ cannot belong to $v X$. 


\section{REFERENCES}

[B] D. K. Burke, A nondevelopable locally compact Hausdorff space with a $G_{8}$-diagonal, General Topology and Appl. 2 (1972), 287-291. MR 47 \#7702.

[G-J] L. Gillman and M. Jerison, Rings of continuous functions, Van Nostrand, Princeton, N.J., 1960. MR 22 \#6994.

[H] N. Hindman, On the existence of c-points in $\beta N \backslash N$, Proc. Amer. Math. Soc. 21 (1969), 277-280. MR 39 \#922.

[P] H. B. Potoczny, A nonparacompact space which admits a closure-preserving cover of compact sets, Proc. Amer. Math. Soc. 32 (1972), 309-311. MR 44 \# 5923.

Department of Mathematics, Carnegie-Mellon University, Pittsburgh, Pennsylvania 15213

Current address: Department of Mathematics, Seton Hill College, Greensburg, Pennsylvania 15601 